PROCEEDINGS OF THE

AMERICAN MATHEMATICAL SOCIETY

Volume 140, Number 1, January 2012, Pages 351-356

S 0002-9939(2011)11041-4

Article electronically published on May 12, 2011

\title{
PLANE CONTINUA AND TOTALLY DISCONNECTED SETS OF BURIED POINTS
}

\author{
JAN VAN MILL AND MURAT TUNCALI
}

(Communicated by Alexander N. Dranishnikov)

\begin{abstract}
It is shown that there is a continuum in the plane whose set of buried points is totally disconnected and weakly 1-dimensional, but not zerodimensional. This answers a problem of Curry, Mayer and Tymchatyn.
\end{abstract}

\section{INTRODUCTION}

For a plane continuum $X$, let $\mathscr{F}(X)$ denote the components of its complement. Put

$$
B(X)=X \backslash \bigcup_{F \in \mathscr{F}(X)} \operatorname{Fr}(F)
$$

Then $B(X)$ is called the set of buried points of $X$. Curry, Mayer and Tymchatyn 2 proved that if $X$ is rim-finite, then $B(X)$ is zero-dimensional, and they constructed an example of a locally connected $X$ for which $B(X)$ is punctiform but not totally disconnected. They asked whether $B(X)$ is zero-dimensional provided it is totally disconnected. The aim of this paper is to answer this question in the negative. Our example is Suslinian but not locally connected, and we do not know whether it is rational.

In [5, Kuratowski presented a 1-dimensional set $K$ in the plane which is zerodimensional at all but countably many points. From the construction, it is clear that this set is totally disconnected and weakly 1-dimensional (see \$4). Our contribution is to show that this set is the set of buried points of some planar continuum.

The sets $B(X)$ are of special interest in case $X$ is the Julia set $J(R)$ of some rational function $R: \mathbb{C}_{\infty} \rightarrow \mathbb{C}_{\infty}$. The question considered in this paper was motivated by the fact that it is unknown whether $B(J(R))$ must be zero-dimensional in case it is punctiform. This is the case for the Devaney-Rocha examples of Sierpińskigasket-like Julia sets. This seems to be a difficult problem. Curry, Mayer and Tymchatyn proposed to study it from a purely topological point of view. For more details, see [1, 2].

Received by the editors November 5, 2010.

2010 Mathematics Subject Classification. Primary 54F15, 54H20, 37B45.

Key words and phrases. Buried point, totally disconnected, plane continuum, zerodimensional, Kuratowski's set.

The first author is pleased to thank the Department of Mathematics and Computer Science at Nipissing University for generous hospitality and support.

The second author is partially supported by National Science and Engineering Research Council of Canada grant No. 141066-2000.

(C)2011 American Mathematical Society Reverts to public domain 28 years from publication 


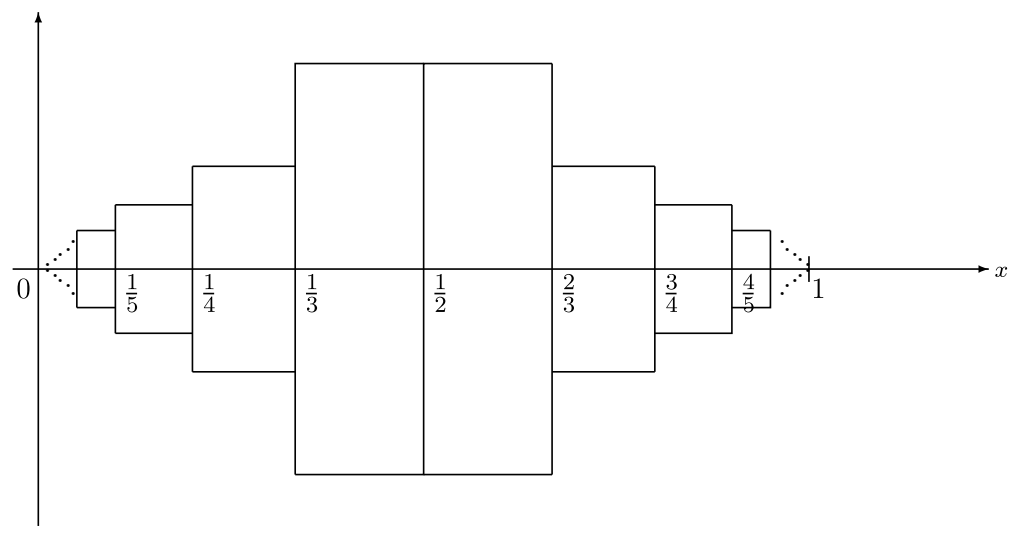

\section{Preliminaries}

If $X$ is a space, then $\operatorname{ind}_{x} X$ denotes the dimension of $X$ at the point $x$; cf. Engelking [4, 1.1.B].

A space is totally disconnected if its quasi-components are points. A space is weakly $n$-dimensional if it is $n$-dimensional but its dimensional kernel is $(n-1)$ dimensional; cf. [4, 1.5.C].

A compact space is rational if it has a basis with countable boundaries.

Let $C$ denote the Cantor set in the closed unit interval $\mathbb{I}$.

If $A$ is a subset of a space $X$, then $\operatorname{Fr}(A)$ denotes its boundary.

For all undefined terms, we refer to Engelking [4].

\section{BURIED POINTS}

In this section we will prove that the set of buried points of a plane continuum can be a Cantor set.

Proposition 3.1. There is a rational locally connected plane continuum $X$ having the following properties:

(1) the boundary of every element of $\mathscr{F}(X)$ is a simple closed curve,

(2) $B(X)$ is a Cantor set.

Proof. Fix $\varepsilon>0$ and, for $n \geq 1$, put

$$
L_{n}=\left[\frac{1}{n+2}, \frac{1}{n+1}\right] \times\left[-\frac{\varepsilon}{n}, \frac{\varepsilon}{n}\right], \quad R_{n}=\left[1-\frac{1}{n+1}, 1-\frac{1}{n+2}\right] \times\left[-\frac{\varepsilon}{n}, \frac{\varepsilon}{n}\right],
$$

respectively.

Then

$$
\triangle=\{(0,0),(1,0)\} \cup \bigcup_{n=1}^{\infty} L_{n} \cup \bigcup_{n=1}^{\infty} R_{n}
$$

is topologically a disc which is contained in the open ball about $\mathbb{I} \times\{0\}$ with radius $3 \varepsilon$.

Observe that if $Z$ is a continuum in $\mathbb{R}^{2}$ which contains

$$
\{(0,0),(1,0)\} \cup \bigcup_{n=1}^{\infty} \operatorname{Fr}\left(L_{n}\right) \cup \bigcup_{n=1}^{\infty} \operatorname{Fr}\left(R_{n}\right)
$$


but misses

$$
\bigcup_{n=1}^{\infty}\left(L_{n} \backslash \operatorname{Fr}\left(L_{n}\right)\right) \cup \bigcup_{n=1}^{\infty}\left(R_{n} \backslash \operatorname{Fr}\left(R_{n}\right)\right),
$$

then $(0,1) \times\{0\}$ does not contain any buried point from $Z$.

Now consider the closed intervals $\left[\frac{1}{3}, \frac{2}{3}\right],\left[\frac{1}{9}, \frac{2}{9}\right],\left[\frac{7}{9}, \frac{8}{9}\right], \ldots$ the interiors of which were removed from $\mathbb{I}$ to obtain $C$. List them faithfully as $\left\{\mathbb{I}_{n}: n \in \mathbb{N}\right\}$. For every $n$ we do the same construction on $\mathbb{I}_{n} \times\{0\}$ with $\varepsilon=\frac{1}{n}$ as we did on $\mathbb{I} \times\{0\}$, thus obtaining a disc $\triangle_{n}$. Clearly, $\triangle_{n} \cap \triangle_{m}=\emptyset$ if $n \neq m$, and the $\triangle_{n}$ form a null-sequence.

The space

$$
S=(C \times\{0\}) \cup \bigcup_{n=1}^{\infty} \triangle_{n}
$$

is a continuum. It is topologically not a disc, since every point from $C \times\{0\}$ is a cut-point of $S$. For every $n$ let $\mathscr{L}_{n}$ denote the collection of interiors of the rectangles that were used to create $\triangle_{n}$. Observe that the boundary of an arbitrary element of $\mathscr{L}_{n}$ is a simple closed curve.

It is not difficult to see that there exists a sequence of disks $D_{1}, D_{2}, \ldots$ in $\mathbb{R}^{2}$ such that $D_{n+1}$ is contained in the interior $E_{n}$ of $D_{n}$ for every $n$, while moreover $\bigcap_{n=1}^{\infty} D_{n}=S$. Consider for a fixed $n$ the set $E_{n} \backslash D_{n+1}$. Its boundary consists of two 'concentric' simple closed curves. Hence we can connect these boundaries by a finite number of arcs $\mathscr{I}_{n}$ such that the collection of components $\mathscr{C}_{n}$ of $\left(E_{n} \backslash D_{n+1}\right) \backslash \bigcup \mathscr{I}_{n}$ has mesh less than $\frac{1}{n}$. We can easily arrange things so that the boundary of every element of $\mathscr{C}_{n}$ is a simple closed curve. Consider the collection of connected open subsets

$$
\mathscr{E}=\left\{\mathbb{R}^{2} \backslash D_{1}\right\} \cup \bigcup_{n=1}^{\infty} \mathscr{C}_{n} \cup \bigcup_{n=1}^{\infty} \mathscr{L}_{n}
$$

of $\mathbb{R}^{2}$. Then $X=\mathbb{R}^{2} \backslash \bigcup \mathscr{E}$ is a locally connected rational continuum such that $\mathscr{F}(X)=\mathscr{E}$ and $B(X)=C \times\{0\}$.

\section{The Construction}

We will now construct a plane continuum $Z$ for which $B(Z)$ is totally disconnected but not zero-dimensional. The continuum is not locally connected, but it has the property that for every $U \in \mathscr{F}(Z)$ we have that $\operatorname{Fr}(U)$ is a simple closed curve. The continuum $Z$ is Suslinian but not locally connected, and we do not know whether it is rational.

Since our construction strongly depends on the geometry of the space $K$, we begin by sketching its construction. See [3, 5] and [4, 1.2.E] for additional details.

Every $x \in C$ can be written uniquely in the form

$$
x=\sum_{i=1}^{\infty} \frac{2 x_{i}}{3^{i}}
$$

for some $\left(x_{1}, x_{2}, \ldots\right) \in\{0,1\}^{\infty}$. For $x \in C \backslash\{0\}$, let $i(x)_{1}<i(x)_{2}<\cdots$ be the sequence (finite or infinite) of all indices $i$ such that $x_{i}=1$. Define a function $f: C \rightarrow[-1,1]$ by

$$
f(x)= \begin{cases}0 & (x=0) \\ \frac{(-1)^{i(x)} 1}{2}+\frac{(-1)^{i(x)}}{2^{2}}+\cdots & (x \neq 0) .\end{cases}
$$


Let $C_{0}$ be the set of all $x \in C \backslash\{0\}$ for which the sequence $i(x)_{1}, i(x)_{2}, \ldots$ is infinite, and let $C_{1}=C \backslash C_{0}$. It is not difficult to verify the following statements:

(K1) $C_{1}$ consists of 0 and the right end-points of the intervals

$$
\left(\frac{1}{3}, \frac{2}{3}\right),\left(\frac{1}{9}, \frac{2}{9}\right),\left(\frac{7}{9}, \frac{8}{9}\right), \ldots
$$

removed from $\mathbb{I}$ to obtain $C$.

(K2) $f$ is continuous at all points of $C_{0}$.

For every $x \in C$, let

$$
T_{x}=\bigcap\{\overline{f(U)}: U \text { is an arbitrary neighborhood of } x \text { in } C\} .
$$

We then have

(K3) For every $x=\frac{2}{3^{i(x)_{1}}}+\frac{2}{3^{i(x)_{2}}}+\cdots+\frac{2}{3^{i(x)_{k}}} \in C_{1} \backslash\{0\}$,

$$
T_{x}=\left[f(x)-\frac{1}{2^{k}}, f(x)+\frac{1}{2^{k}}\right] .
$$

Moreover,

$$
T_{0}=[-1,1] .
$$

Let $K=\{(x, f(x)): x \in C\}$ be the graph of the function $f$. The following is not trivial; see [3] and the hint in [4, 1.2.E(e)] .

(K4) $\operatorname{ind}_{(x, f(x))} K=0$ if $x \in C_{0}$ and $\operatorname{ind}_{(x, f(x))} K=1$ if $x \in C_{1}$.

Hence $K$ is 1-dimensional precisely at the countably many points of the form $(x, f(x))$, where $x \in C_{1}$.

The following statements are again not difficult to check:

(K5) The set

$$
D=K \cup \bigcup_{x \in C_{1}}\left(\{x\} \times T_{x}\right) \subseteq \mathbb{R}^{2}
$$

is compact. Moreover, $K$ is a $G_{\delta}$-subset of $D$ (and hence is Polish).

(K6) $K$ is totally disconnected and weakly 1-dimensional.

We are now ready to construct the example. To begin with, we slightly enlarge $D$. For every $x=\frac{2}{3^{i(x)_{1}}}+\frac{2}{3^{i(x)_{2}}}+\cdots+\frac{2}{3^{i(x)_{k}}} \in C_{1} \backslash\{0\}$, we put

$$
s_{x}=x-\frac{1}{2 \cdot 3^{i(x)_{k}}} \text {. }
$$

Moreover, $s_{0}=-\frac{1}{2}$. It is clear that $\left[s_{x}, x\right] \cap C=\{x\}$ for every $x \in C_{1}$. Observe that the collection of intervals

$$
\mathscr{B}^{\prime}=\left\{\left[s_{x}, x\right]: x \in C_{1}\right\}
$$

is a pairwise disjoint null-sequence. Hence for every $\varepsilon>0$ there are only finitely many rectangles of the form $\left[s_{x}, x\right] \times T_{x}$ for $x \in C_{1}$ that are not contained in the $\varepsilon$-ball about the compact set $D$. This clearly implies that the set

$$
E=K \cup \bigcup_{x \in C_{1}}\left(\left[s_{x}, x\right] \times T_{x}\right) \subseteq \mathbb{R}^{2}
$$

is compact.

Put

$$
\mathscr{B}=\left\{\left[s_{x}, x\right] \times T_{x}: x \in C_{1}\right\}
$$



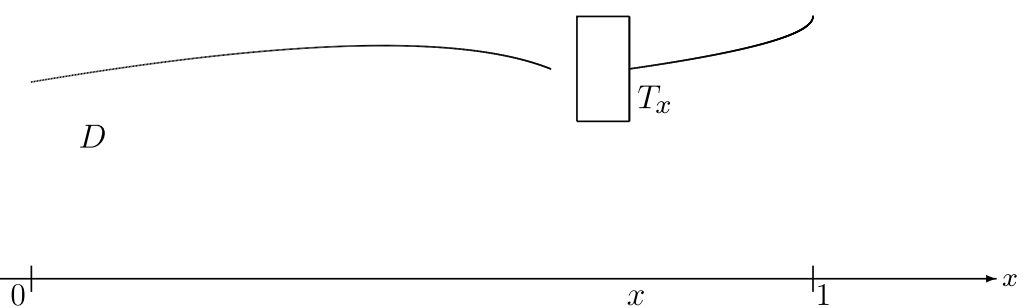

and observe that $\mathscr{B}$ coincides with the collection of all nondegenerate components of $E$. Since the collection of components of a compact space is an upper semicontinuous decomposition of that space, it is clear that the decomposition $\mathscr{D}$ of $\mathbb{R}^{2}$, the nondegenerate members of which are the elements of $\mathscr{B}$, i.e.,

$$
\mathscr{D}=\mathscr{B} \cup\left\{\{z\}: z \in \mathbb{R}^{2} \backslash \bigcup \mathscr{B}\right\},
$$

is upper semicontinuous. Let $\pi: \mathbb{R}^{2} \rightarrow \mathbb{R}^{2} / \mathscr{D}$ be the corresponding quotient map. By Moore's Theorem from [7, $Y=\mathbb{R}^{2} / \mathscr{D}$ is homeomorphic to $\mathbb{R}^{2}$. It is clear that $\pi(E)$ is a Cantor set. Observe that all Cantor sets in $\mathbb{R}^{2}$ are topologically equivalent within $\mathbb{R}^{2}$ (Kuratowski [6, p. 537]). Hence by Proposition 3.1, there is a continuum $X$ in $Y$ and a pairwise disjoint collection $\mathscr{U}$ of connected open subsets of $Y$ such that

(1) $Y \backslash \bigcup \mathscr{U}=X$,

(2) $\operatorname{Fr}(U)$ is a simple closed curve for every $U \in \mathscr{U}$,

(3) $X \backslash \bigcup_{U \in \mathscr{U}} \operatorname{Fr}(U)=\pi(E)$ (hence $B(X)=\pi(E)$ ).

Since $\pi$ is monotone, $X^{\prime}=\pi^{-1}(X)$ is a subcontinuum of $\mathbb{R}^{2}$. Also, the nondegeneracy set of $\pi$ is contained in $\pi(E)$. By abuse of notation, we will identify $\mathbb{R}^{2} \backslash E$ and $Y \backslash \pi(E)$. Observe that under this identification, $\mathscr{U}$ is a pairwise disjoint collection of connected open subsets of $\mathbb{R}^{2}$ such that

(4) $\mathbb{R}^{2} \backslash \bigcup \mathscr{U}=X^{\prime}$,

(5) $\operatorname{Fr}(U)$ is a simple closed curve for every $U \in \mathscr{U}$,

(6) $X^{\prime} \backslash \bigcup_{U \in \mathscr{U}} \operatorname{Fr}(U)=E$ (hence $B(X)=E$ ).

Fix an arbitrary $x \in C_{1}$, and consider the rectangle $\left[s_{x}, x\right] \times T_{x}$. Recall that $T_{x}=\left[f(x)-\frac{1}{2^{k}}, f(x)+\frac{1}{2^{k}}\right]$ for certain $k \in \mathbb{N}$. It is geometrically obvious that there is a collection $\mathscr{V}_{x}$ of pairwise disjoint connected open subsets of $\left(s_{x}, x\right) \times$ $\left(f(x)-\frac{1}{2^{k}}, f(x)+\frac{1}{2^{k}}\right)$ such that

(7) $\operatorname{Fr}(V)$ is a simple closed curve for every $V \in \mathscr{V}$,

(8) $\bigcup_{V \in \mathscr{V}_{x}} \bar{V}=\left(\left[s_{x}, x\right] \times T_{x}\right) \backslash\{(x, f(x))\}$.

It is clear that $\bigcup_{V \in \mathscr{V}_{x}} \operatorname{Fr}(V)$ is connected.

Now consider the collection

$$
\mathscr{W}=\mathscr{U} \cup \bigcup_{x \in C_{1}} \mathscr{V}_{x}
$$

It consists of connected open subsets of $\mathbb{R}^{2}$ with simple closed curves as boundaries. Also, $Z=\mathbb{R}^{2} \backslash \bigcup \mathscr{W}$ is bounded and hence compact. Since $X^{\prime}$ is a continuum, $\operatorname{Fr}\left(\left[s_{x}, x\right] \times T_{x}\right)$ meets the closure of $X^{\prime} \backslash\left(\left[s_{x}, x\right] \times T_{x}\right)$ and hence the connected set $\bigcup_{V \in \mathscr{Y}_{x}} \operatorname{Fr}(V)$ for every $x \in C_{1}$. This clearly implies that $Z$ is a continuum. From the construction it is clear that $B(Z)=K$. 


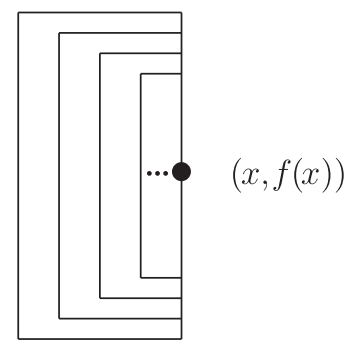

We will now show that $Z$ is Suslinian. Observe that $Z$ is $D$ with a countable collection $\mathscr{I}=\left\{\mathbb{I}_{n}: n \in \mathbb{N}\right\}$ of arcs added to it. Let $\mathscr{M}$ be a collection of pairwise disjoint nondegenerate subcontinua of $Z$. A moment's reflection shows that only countably many members from $\mathscr{M}$ are contained in $D$. Since $\mathscr{M}$ consists of continua and every nonempty open subset of a continuum contains a nontrivial continuum, we may consequently assume without loss of generality that every member from $\mathscr{M}$ misses $D$. If $M \in \mathscr{M}$, then $\mathbb{I}_{n} \cap M$ is not zero-dimensional for every $n$ since otherwise $M$ would be zero-dimensional, [4, 1.3.1]. Hence for every $M \in \mathscr{M}$ there exists $n \in \mathbb{N}$ such that $\mathbb{I}_{n} \cap M$ contains a nontrivial interval from $\mathbb{I}_{n}$. This clearly implies that $\mathscr{M}$ is countable.

\section{REFERENCES}

[1] C. P. Curry and J. C. Mayer, Buried points in Julia sets, Journal of Difference Equations and Applications 16 (2010), 435-441. MR.2642460

[2] C. P. Curry, J. C. Mayer, and E. D. Tymchatyn, Buried points in rational Julia sets, Spring Topology and Dynamics Conference, Miss. State U., March 18, 2010.

[3] Alan Dow and Klaas Pieter Hart, Cosmic dimensions. Topology Appl. 154 (2007), no. 12, 2449-2456. MR2333799 (2008j:54026)

[4] R. Engelking, Theory of dimensions finite and infinite, Heldermann Verlag, Lemgo, 1995. MR.1363947 (97j:54033)

[5] K. Kuratowski, Une application des images de fonctions à la construction de certains ensembles singuliers, Mathematika 6 (1932), 120-123.

[6] K. Kuratowski, Topology. II, Academic Press, New York, 1968. MR0259835 (41:4467)

[7] R. L. Moore, Concerning upper semi-continuous collections of continua, Trans. Amer. Math. Soc. 27 (1925), 416-428. MR1501320

Department of Mathematics, Faculty of Sciences, VU University Amsterdam, De Boelelaan 1081, 1081 HV Amsterdam, The Netherlands

E-mail address: vanmill@few.vu.nl

Department of Computer Science and Mathematics, Nipissing University, 100 College Drive, North Bay, ON, P1B 8L7, Canada

E-mail address: muratt@nipissingu.ca 